\title{
MONOLAYERS AT LIQUID INTERFACES
}

\begin{abstract}
$\mathrm{A}^{\mathrm{N}}$ informal discussion of the Faraday Society on monolayers at liquid interfaces was held at Loughborough College of Technology during September 9-10, 1965. The meeting was attended by 68 people, among whom there was strong representation from European research laboratories. There were 27 from industrial organizations.

The chair for the session on September 9 was taken by Dr. J. L. Moilliet (Imperial Chemical Industries, Dyestuff Division). After a brief welcome from Prof. R. F. Phillips (Loughborough College of Technology) the opening paper was given by Prof. G. M. Bell (Chelsea College of Science and Technology) and Dr. S. Levine (University of Manchester). This was concerned with the surface-free energy and the surface pressure of monolayers. The relation between the part of the surface Helmholtz-free energy, $F$, due to dissolved components and surface-active species, and the corresponding part of the surface pressure, II, was discussed. When all surface-active substances are soluble in the substrate, and $F$ is defined as the change in surface-free energy density during a process in which they are brought into the surface region from the bulk of the substrate, then it was shown that $\Pi=-F$. This is not true if any of the surface-active substances are insoluble and an expression for $\mathrm{II}+F$ was obtained in this case. The form of II obtained in this way was shown to be identical with that derived by integration of the Gibbs adsorption isotherm (as used by Onsager and Samaras and others for air-electrolyte interfaces). The authors concluded with a brief mention of the various contributions to the spreading pressure of an insoluble partially ionized monolayer on an aqueous electrolyte substrate.
\end{abstract}

The second paper was given by Drs. J. Mingins, J. W. Brooks and B. A. Pethica (Unilever Research Laboratory, Port Sunlight) and described investigations of ionized monolayers. Previous data on the surface pressures of insoluble and soluble ionized monolayers at both nonpolar oil-water and air-water interfaces were critically reviewed and compared with recent data obtained at Port Sunlight. Discrepancies among the data were discussed in terms of solution of the monolayer, poor spreading, and impurities in the surface-active agents, electrolytes and water. Results on films spread from crystals indicated that solvent retention had occurred in some of the earlier published work on gaseous monolayers spread at the air-water interface. The cohesive pressure due to hydrocarbon chain interaction was shown to be negligibly small at non-polar oil-water interfaces over the range of areas per molecule studicd $\left(50 \AA^{2}\right)$. However, at air-water interfaces the cohesive pressure was a complex function of chain-length, area per molecule and head group. The validity of previous empirical equations describing this term was questioned.

Dr. E. H. Lucassen-Reynders (Unilever, Vlaardingen) presented a thermodynamic approach to the surface equation of state. This included consideration of ionizing surfactants and non-surface active electrolytes. The resulting equations are similar to those for non-ionizing surfactants, except that the molar concentration in the solution is replaced by the geometric mean of the product of the concentrations of the long-chain ions and counterions. The general equations give the surface tension as a function of the mean ionic product in terms of three types of parameters. These are, first, the interfacial activity coefficients, $\gamma_{i}^{s}$, of the surface mixture of surfactant and water; secondly, the saturation adsorption, $\Gamma^{\infty}$ (the total values for long-chain ions and counter-ions); and thirdly, a parameter, $a$, measuring the free adsorption enthalpy at infinite dilution. The equations were applied to published surface and interfacial tension measurements on adsorbed and spread monolayers of long-chain alkylsulphates, trimethyl ammonium bromides and carboxylates, in the presence of various amounts of non-surfaceactive electrolytes. At oil-water interfaces, the ionized surfactants were shown to form ideal surfaces $\left(\gamma_{i}^{\delta}=1\right)$, irrespective of the presence of inorganic electrolyte with a common ion. At the air-water interfaces especially the longer-chain surfactants were shown not to form ideal surfaces. In particular, carboxylate soap solutions show regular surface behaviour. The $\gamma_{i}^{s}$ values obtained from the experimental data suggested that the main factor responsible for the non-ideality of a surface was the interaction between the hydrophobic chains of the surfactant.

A further paper on the thermodynamics of interfaces was presented by Dr. A. J. Spaull (Brunel College). Methods for obtaining thermodynamic parameters for adsorption at the air-water interface were considered. It was maintained that the initial linear region of the adsorption isotherm is important, but it was suggested that use of the slope, $\alpha$, of the plot of surface pressure as a function of concentration, can lead to incorrect values of the thermodynamic parameters. Analysis has shown that, to first order, the plot of the ratio of surface pressure to concentration as a function of surface pressure can be represented by the equation:

$$
\Pi / c_{x}=\left(\Pi / c_{x}\right)_{0}(\mathrm{I}-a / R T)
$$

where $a$ is a two-dimensional second virial coefficient, and $\left(11 / c_{x}\right)_{0}$ is the extrapolated value of the ratio of surface pressure to concentration at zero surface pressure. To calculate correct thermodynamic parameters it is $\left(\mathrm{II} / c_{x}\right)_{1}$ rather than $\alpha$ that is required, except when $a$ is zero. Experimental methods for testing this argument were indicated. Certain thermodynamic parameters obtained for the adsorption of 1-butanol and 1-hexanol were used to test a recent theory of Aranow and Witten, who claim that adsorption of long-chain hydrocarbons is essentially an entropic process arising from restricted rotation about carbon bonds in aqueous solution. Some difficulties of the theory in explaining the evidence of the nuclear magnetic resonance spectra of alcohol solutions and the solvation of rare gases were discussed.

Dr. J. B. Matthews (Loughborough College of Technology) proposed a new approach to the dynamics of adsorption from solution The kineties of adsorption from solution is still a controversial subject, owing to the uncertainties associated with the experimental data and the model for the adsorption process. A number of theories for the kinetics of adsorption have culminated in the Ward-Tordai theory, which now receives general acceptance, either in its original form or as modified by others. The model proposed by Ward and Tordai assumes the existence of a sub-surface from which solute molecules are adsorbed instantaneously and irreversibly to the surface, if there are available sites for them. The kinetics of adsorption are assumed to be controlled by the diffusion of solute molecules to the sub-surface from the bulk of the solution. Dr. Matthews stated that this model ignores the dynamic character of adsorption and transitions between the possible states of adsorbed molecules. The mathematical treatment of this model leads to kinetic results which are more consistent with experimental data than the Ward and Tordai theory. 
The chairman for the second day was Prof. D. D. Eley (University of Nottingham). In opening the scssion Prof. Eley paid tribute to Dr. J. J. Kipling, who was a member of the organizing committee of the discussion and whose untimely death occurred at a conference in Moscow earlier in the year.

The first paper was given by Prof. A. Scheludko (Institute of Physical Chemistry, Sofia, Bulgaria), who reported work carried out with Dr. D. 'Tissen on the damping of ripples on water by soluble surfactants and insoluble monolayers. A novel apparatus has been developed for this problem, consisting of a cylindrical vessel in which standing waves are generated by a vertical sinusoidal vibration. The amplitude of ripples smaller than $30 \mu$ could be determined optically with an accuracy of 1 per cent and the damping measured by changing the energy supplied to the vessel. Results wore presented for the damping coefficient as a function of concentration (or area) for caproic, capric, myristic, palmitic and propyldodecyl acetic acids and for cetyl alcohol on water. With all except the first, a sharp maximum was found at intermediate surface densities and damping coefficients at the maximum exceeded (for a factor of $2-3$ ) the value predicted by the hydrodynamic theory of Levich for damping by a condensed monolayer.

The noxt paper, by Drs. J. M. Corkill, J. F. Goodman, S. P. Harrold and J. R. Tate (Proctor and Gamble, Newcastle upon Tyne), was concerned with mixed monolayers of both soluble and insoluble salts of the type alkyltrimethylammonium-alkyl-sulphate at the air-solution interface. Insoluble monolayers were examined using a Langmuir trough. It was demonstrated that although second-order effects are present due to the substrate or the particular molecular species involved, maximum interaction invariably occurs at a $1: 1$ ratio of long-chain cation to anion. The composition of the surfaco layers of the soluble members was measured by radio-tracer techniques. When the long-chain cation and anion had the same chain-length an equimolar surface composition was obtained. However, if the ions had different chainlengths, the longer chain ion was preforentially adsorbed giving an asymmetric surface layer, the asymmetry being enhanced by the addition of inorganic electrolytes. It was concluded that adsorption is promoted principally by the elimination of alkyl chain-water interface, but this is modified by the electrical interaction between the positive and negative hoad groups, which is a maximum when the surface composition is equimolar.

A paper by Dr. J. J. Kipling and Dr. C. A. J. Langman (University of Hull) dealt with the surface tension of two component liquid mixtures and the adsorption from these mixtures at the liquid-vapour and liquid-solid interfaces. The surface tensions of a number of binary liquid mixtures of molecules of similar sizes were measured and the results were shown to fit quite well an equation based on a regular solution model, relating surface tension to composition. The adsorption from the mixtures at low-energy solid surfaces was studied and the surface excess of the more strongly adsorbed molecule compared with the corresponding surface oxcess at the liquid-vapour interface. In all cases presented, these two excesses were similar in magnitude.

Another paper on the surface tensions of binary liquid mixtures, by Dr. R. Aveyard (University of Cambridge), concluded the conference. The surface tensions of a range of two-component mixtures of $n$-alkanes have been measured. The applicability of various equations to the experimental data was tested and it was found that a theory, devised by Prigogine and Maréchal for athermal polymer solutions, was the most satisfactory of those considered. Using this theory, the surface tensions of all the mixtures investigated could be predicted to within 1.0 per cent with the use of physically realistic and selfconsistent parameters. Further, the applicability of this theory to the surface tensions of methanol- $n$-decanol and methanol-glycerol mixtures was tested. The agreement between experiment and theory was poor for the former system, but reasonable for the latter. A possible explanation for theso differences, and its bearing on the agreement obtained for the $n$-alkane systems, was discussed.

D. A. HAYDON

M. J. JAYCOOK

\section{PHYSICS IN MEDICINE}

T ECHNOLOGY, rather than science, was the main theme of the first International Conference on Medical Physics, held at Harrogate during September 8-10, but several topics of a more fundamental kind were also illuminated in a programmo of more than 100 papers. A majority of the contributions dealt with the elinical and biological applications of ionizing radiations, reflecting a continuing preoccupation with the problems which brought physicists into tho hospital service in significant numbers some 30 years ago.

Though the radiotherapist's armamentarium has not changed substantially in recent years, two areas of research at present give promise of greater accuracy in the measurement and control of radiation dosage for clinical purposes. The first problem here is to find a device of small size (preferably in the millimetro range) with a sonsitive response independent of radiation energy. Lithium fluoride dosemeters, based on the thermoluminescont effect, satisfy these requiroments reasonably woll. Largescale practical applications are, however, restrained by inadequate understanding of the basic processes involved. The preparation of lithium fluoride powder and its treatment before and after irradiation are still largely determined by empirical knowledge, which necessitates caution in the interpretation of experimental findings.

Thermolumineseent dosemeters have been used for the estimation of surface dosage in radiotherapy, but not so far for internal measurements. F. W. Spiers (General
Infirmary, Leeds) described methods for tho study of radiation dose in specimens of dry bone, using fine-grain lithium fluoride powder which is introduced into the narrow cavities of trabecular bone and removed for assay after irradiation. Work of this kind is usoful in assessing clinical, occupational and environmental hazards from radioactive materials. B. E. Bjarngard (Controls for Radiation, Inc., Cambridge, Massachusetts) reported that some of the difficulties associated with the preparation and handling of thermoluminescent powders may be avoided by incorporating the lithium fluoride phosphor in polytetrafluorethylene, which can then be fashioned into rods, disks or foils for various applications. The combination has an effective atomic number close to that of tissue, and its response is independent of radiation energy over a fairly wide range.

Interesting applications are now being found for solid state devices as radiation detectors. P. R. Parker (Institute of Cancer Research, Sutton, Surrey) suggested that many of the desirable properties of the gas-filled ionization chamber may be reproduced, with the advantage of very small bulk, by $p-n$ surface barrier detectors. Used as pulse counters, these devices will measure dose-rates of about $10 \mu \mathrm{rad} / \mathrm{h}$, approximately equal to the natural background radiation in Britain. The radiation-induced potential across the $p-n$ junction is found to be a logarithmic function of dose-rate and the short-circuit current is approximately a linear function of dose-rate. Silicon 